\title{
AN ALGEBRAIC PROOF OF A THEOREM OF A. ROBINSON ${ }^{1}$
}

\author{
ROBERT GILMER AND JOE L. MOTT
}

\begin{abstract}
A. Robinson has used mathematical logic to obtain a theorem concerning systems of polynomial equations with only finitely many solutions; this paper contains an algebraic proof of Robinson's theorem, based primarily on various equivalent forms of Hilbert's Nullstellensatz.
\end{abstract}

In $[4$, p. 38], Robinson uses principles of logic to solve some problems in algebra. By use of lower predicate calculus and Gödel's completeness theorems $[4$, p. 12], he gives elegant proofs of some known results and at least one new result; Robinson indicates that this new result has no readily accessible purely mathematical solution independent of his approach.

Statement of the Theorem. Let $f_{1}, \cdots, f_{t}$ be polynomials in $n$ indeterminates with integer coefficients (that is, $f_{1}, \cdots, f_{t}$ $\left.\in Z\left[X_{1}, \cdots, X_{n}\right]\right)$ and denote by $\bar{f}_{i}^{p}$ the polynomial which is obtained from $f_{i}$ by reducing its coefficients modulo $p$. If the system of equations $f_{1}=0, f_{2}=0, \cdots, f_{t}=0$ has at most $m$ solutions $i n$ any extension field of the rationals, then there is a prime $q$ such that for each prime $p>q$, the system $\bar{f}_{1}^{p}=0, \bar{f}_{2}^{p}=0, \cdots, \bar{f}_{t}^{p}=0$ has at most $m$ solutions in any extension field of the finite field $Z_{p}$.

While we concede the power and beauty of Robinson's approach, the purpose of this note is to give an elementary algebraic proof of the preceding theorem. Our proof will consist of three parts; the first two parts will be separated in to lemmas.

First we remark that the Hilbert Nullstellensatz, as commonly stated [8, p. 164], asserts that if a polynomial $f\left(X_{1}, \cdots, X_{n}\right)$ over a field $k$ vanishes at every zero of an ideal $A$ of $k\left[X_{1}, \cdots, X_{n}\right]$, then some power of $f$ is in $A$. On the basis of this result (or by use of Zariski's Nullstellensatz [7, pp. 362-363]), one can establish a oneto-one correspondence between the set of maximal ideals of $k\left[X_{1}, \cdots, X_{n}\right]$ and a certain set of equivalence classes in $n$-dimen-

Received by the editors October 8, 1970.

AMS 1970 subject classifications. Primary 14A10; Secondary 13L05.

Key words and phrases. Hilbert's Nullstellensatz, Gödel's completeness theorem, predicate calculus.

1 The authors received support under National Science Foundation Grant GP19406.

Copyright (c) 1971, American Mathematical Society 
sional affine space over the algebraic closure $\bar{k}$ of $k$. The equivalence relation referred to is the following. The points $\left(x_{1}, \cdots, x_{n}\right)$ and $\left(y_{1}, \cdots, y_{n}\right)$ are equivalent if there is a $k$-isomorphism from $k\left(x_{1}, \cdots, x_{n}\right)=k\left[x_{1}, \cdots, x_{n}\right]$ onto $k\left(y_{1}, \cdots, y_{n}\right)=k\left[y_{1}, \cdots, y_{n}\right]$ mapping $x_{i}$ onto $y_{i}$ for each $i$. Using this correspondence, the Nullstellensatz can be reinterpreted to state that the intersection of all maximal ideals which contain $A$ is the radical of $A[2, \mathrm{p} .47]$. This fact will be used frequently in the proof of Lemma 1.

If $F$ is a field, we denote $n$-dimensional affine space over $F$ by $A_{n}^{p}$. If $F$ is an extension field of $k$ and if $B$ is an ideal of $k\left[X_{1}, \cdots, X_{n}\right]$, we recall that the variety of $B$ in $A_{n}^{F}$ is the set of all points $(y)$ $=\left(y_{1}, \cdots, y_{n}\right) \in A_{n}^{p}$ such that $f(y)=0$ for each $f$ in $B$. We shall denote this variety by $V_{F}(B)$.

We make one final remark concerning Hilbert's Nullstellensatz. Another formulation [8, p. 164], due originally to Rabinowitsch [3] asserts that $V_{\vec{k}}(B)$ is empty if and only if $B=k\left[X_{1}, \cdots, X_{n}\right]$. This formulation is used to give a trivial purely mathematical proof of a special case of Robinson's theorem when $m=0$ [4, p. 38]. For this reason, we shall tacitly assume that the ideal $A$ of $k\left[X_{1}, \cdots, X_{n}\right]$ is not equal to $k\left[X_{1}, \cdots, X_{n}\right]$.

Lemma 1. Let $k$ be a field, let $g_{1}, \cdots, g_{t} \in k\left[X_{1}, \cdots, X_{n}\right]$, and let $A$ be the ideal of $k\left[X_{1}, \cdots, X_{n}\right]$ generated by $\left\{g_{i}\right\}_{i=1}^{t}$. Further, suppose that $A \neq k\left[X_{1}, \cdots, X_{n}\right]$. Then the following are equivalent:

(a) $V_{F}(A)$ is finite for every extension field $F$ of $k$.

(b) $V_{\overline{\mathbf{k}}}(A)$ is finite, where $\bar{k}$ is the algebraic closurc of $k$.

(c) The set of maximal ideals of $k\left[X_{1}, \cdots, X_{n}\right]$ containing $A$ is finite and nonempty.

(d) The ideal $A$ is of depth zero. ${ }^{2}$

(e) The ideal $A F\left[X_{1}, \cdots, X_{n}\right]$ is of depth zero for any extension field $F$ of $k$.

Lemma 1 is known, but a precise reference in the literature is difficult to find. For example, it follows from our discussion above that $(\mathrm{a}) \rightarrow(\mathrm{b}) \rightarrow(\mathrm{c})$, and the implication $(\mathrm{c}) \rightarrow(\mathrm{d})$ is obvious. The implication (e) $\rightarrow$ (a) follows from the equalities $V_{\bar{F}}(A)=V_{\bar{P}}(\sqrt{ } A)$, $V_{\bar{P}}\left(\bigcap_{i=1}^{n} M_{i}\right)=\bigcup_{i=1}^{n} V_{\bar{P}}\left(M_{i}\right)$, and $\left|V_{\bar{P}}(M)\right|=\left[F\left[X_{1}, \cdots, X_{n}\right] / M: F\right]_{8} ;$ here $[K: F]_{s}$ denotes the separable degree of the field $K$ over its sub-

\footnotetext{
2 We say that an ideal $A$ of a ring $R$ is of depth zero if $A \neq R$ and if the only prime ideals of $R$ containing $A$ are maximal. In particular, since $k\left[X_{1}, \cdots, X_{n}\right]$ is Noetherian, where $k$ is a field, an ideal $A$ of $k\left[X_{1}, \cdots, X_{n}\right]$ of depth zero is contained in only finitely many maximal ideals.
} 
field $F$ (where $K / F$ is algebraic), $|S|$ denotes the cardinality of the set $S$, and $M$ denotes a maximal ideal of $F\left[X_{1}, \cdots, X_{n}\right][5$, pp. 5657]. That $(d) \rightarrow(e)$ follows from Proposition 3 of $[6$, p. 74], but for the proof of Lemma 2, we will need an analysis of the implication (d) $\rightarrow(\mathrm{e})$.

We observe that, in general, if a ring $S$ is integral over a ring $R$ and if $A$ is an ideal of $R$, then $A$ has depth zero if and only if $A S$ has depth zero $\left[1\right.$, p. 31]. Therefore, the ideal $A F\left[X_{1}, \cdots, X_{n}\right]$ of $F\left[X_{1}, \cdots, X_{n}\right]$ will be of depth zero if and only if $A \bar{F}\left[X_{1}, \cdots, X_{n}\right]$ has depth zero, where $\bar{F}$ is the algebraic closure of $F$. Thus, we may assume that $k$ and $F$ are algebraically closed fields. Since $k$ is algebraically closed, each maximal ideal of $k\left[X_{1}, \cdots, X_{n}\right]$ is of the form $M=\left(X_{1}-\alpha_{1}, \cdots, X_{n}-\alpha_{n}\right)$ for some $\alpha_{i} \in k[8$, p. 198]. Clearly $M F\left[X_{1}, \cdots, X_{n}\right]$ is a maximal ideal of $F\left[X_{1}, \cdots, X_{n}\right]$. Thus there is a unique maximal ideal of $F\left[X_{1}, \cdots, X_{n}\right]$ containing $M$. If $P$ is a proper prime ideal of $F\left[X_{1}, \cdots, X_{n}\right]$ containing $A$, then $P^{\prime}=$ $P \cap k\left[X_{1}, \cdots, X_{n}\right]$ is a prime ideal of $k\left[X_{1}, \cdots, X_{n}\right]$ containing $A$. Thus $P^{\prime}$ is a maximal ideal, $P^{\prime} F\left[X_{1}, \cdots, X_{n}\right] \subseteq P$, and $P^{\prime} F\left[X_{1}, \cdots, X_{n}\right]$ a maximal ideal of $F\left[X_{1}, \cdots, X_{n}\right]$ imply that $P^{\prime} F\left[X_{1}, \cdots, X_{n}\right]=P$ and $A F\left[X_{1}, \cdots, X_{n}\right]$ is of depth zero.

In the above discussion, we observed a one-to-one correspondence between the set of maximal ideals of $\bar{k}\left[X_{1}, \cdots, X_{n}\right]$ containing $A_{\overline{\boldsymbol{k}}}$ (we continue to use $A$ to denote the ideal of $k\left[X_{1}, \cdots, X_{n}\right]$ generated by $\left\{g_{i}\right\}_{i=1}^{t}$, and by $A_{U}$ the ideal $A U\left[X_{1}, \cdots, X_{n}\right]$ for any extension field $U$ of $k$ ) and the set of maximal ideals of $\bar{F}\left[X_{1}, \cdots, X_{n}\right]$ containing $A_{\bar{F}}$. Hence, if $V_{\bar{k}}(A)$ is finite, then $\left|V_{F}\left(A_{F}\right)\right| \leqq\left|V_{\bar{F}}\left(A_{\bar{F}}\right)\right|$ $=\left|V_{\overline{\mathbf{k}}}(A)\right|$, so that the number of solutions in $A_{n}^{F}$ is always less than or equal to the number of solutions in $A_{n}^{\bar{k}}$, where $F$ is any extension field of $k$.

Now if the ideal $A$ of $k\left[X_{1}, \cdots, X_{n}\right]$ is of depth zero, then $\sqrt{ } A=M_{1} \cap \ldots \cap M_{s}$, where $M_{i}$ is a maximal ideal, and $k\left[X_{1}, \cdots, X_{n}\right] / \sqrt{ } A$ is a vector space over $k$ which is isomorphic to the direct sum of its subspaces $\left\{k\left[X_{1}, \cdots, X_{n}\right] / M_{i}\right\}_{i=1}^{s}$. Therefore the dimension of $k\left[X_{1}, \cdots, X_{n}\right] / \sqrt{ } A$ over $k$ is the sum of the dimensions of $k\left[X_{1}, \cdots, X_{n}\right] / M_{i}$ over $k$. Since $V_{\bar{k}}(A)=V_{\bar{k}}\left(M_{1}\right) \cup \cdots$ $\cup V_{\bar{k}}\left(M_{\varepsilon}\right)$, it follows that $\left|V_{\bar{k}}(A)\right|=\sum_{i=1}^{s}\left|V_{\bar{k}}\left(M_{i}\right)\right|$. In general,

$$
\left|V_{\bar{k}}\left(M_{i}\right)\right|=\left[k\left[X_{1}, \cdots, X_{n}\right] / M_{i}: k\right]_{s} \leqq \operatorname{dim}_{k}\left(k\left[X_{1}, \cdots, X_{n}\right] / M_{i}\right) .
$$

However, when $k$ is a perfect field, equality holds. We state this result in the next lemma.

LEMMA 2. If $k$ is a perfect field and if $B$ is an ideal of $k\left[X_{1}, \cdots, X_{n}\right]$ 
such that $\sqrt{ } B=M_{1} \cap \cdots \cap M_{s}$, where $M_{i}$ is a maximal ideal for each $i$, then $\left|V_{\bar{k}}(B)\right|=\sum_{i=1}^{s} \operatorname{dim}_{k}\left(k\left\lfloor X_{1}, \cdots, X_{n}\right] / M_{i}\right)$.

We proceed now to the proof of Robinson's theorem. Let $f_{1}, \cdots, f_{t}$ $\in Z\left[X_{1}, \cdots, X_{n}\right]$, and let $A_{Z}$ be the ideal of $Z\left[X_{1}, \cdots, X_{n}\right]$ generated by $\left\{f_{1}, \cdots, f_{t}\right\}$. We assume that $A_{Z} \neq Z\left[X_{1}, \cdots, X_{n}\right]$. If $A_{Q}$ denotes the ideal in $Q\left[X_{1}, \cdots, X_{n}\right]$ generated by $\left\{f_{1}, \cdots, f_{t}\right\}$, then the assumption that $V_{F}\left(A_{Q}\right)$ has at most $m$ elements implies, by Lemma 1 , that $A_{Q}$ is an ideal of depth zero. Since $Q\left[X_{1}, \cdots, X_{n}\right]$ $=\left(Z\left[X_{1}, \cdots, X_{n}\right]\right)_{N}$, where $N=Z-\{0\}$, it follows that any shortest primary decomposition of $A_{Z}$ is of the form $A_{Z}=Q_{1} \cap \cdots \cap Q_{8}$ $\cap Q_{1}^{\prime} \cap \cdots \cap Q_{r}^{\prime}$, where $P_{i}=\sqrt{ } Q_{i}$ is maximal with respect to not meeting $Z-\{0\}$ for each $i$, and $\sqrt{ } Q_{j}^{\prime}=P_{j}^{\prime}$ meets $Z$ in $p_{j}^{\prime} Z \neq(0)$ for each $j$.

Let $p$ be a prime integer not in the set $\left\{p_{1}^{\prime}, \cdots, p_{r}^{\prime}\right\}$. We will show that for any such prime $p$, the system $\bar{f}_{1}^{p}=0, \cdots, \bar{f}_{t}^{p}=0$ over $Z_{p}\left[X_{1}, \cdots, X_{n}\right]$ has at most $m$ solutions in $A_{n}^{F}$, where $F$ is any extension field of $Z_{p}$.

The ideal $A_{p}=\left[A_{Z}+(p)\right] /(p)$ of $Z_{p}\left[X_{1}, \cdots, X_{n}\right]$ is generated by $\bar{f}_{1}^{p}, \cdots, \bar{f}_{t}^{p}$. We wish to show that $\left|V_{\bar{Z}_{p}}\left(A_{p}\right)\right| \leqq\left|V_{\bar{Q}}\left(A_{Q}\right)\right|$. Thus we can assume that $A_{p} \neq Z_{p}\left[X_{1}, \cdots, X_{n}\right]$. Consider the radical of $A_{Z}+(p)$ in $Z\left[X_{1}, \cdots, X_{n}\right]$. If $P$ is a prime ideal containing $A_{Z}+(p)$, then $P \supseteq P_{i}$ for some $i$ or $P \supseteq P_{j}^{\prime}$ for some $j$. Since $p \in P$ and $p_{j}^{\prime} \in P_{\jmath}^{\prime}$, we see that $P \supseteq P_{i}$ for some $i$. Since $P_{i}$ extends to a maximal ideal of $Q\left[X_{1}, \cdots, X_{n}\right]$, it follows that $P_{i}^{e}$ is of height $n$ in $Q\left[X_{1}, \cdots, X_{n}\right]$ $\left[8\right.$, p. 193]. ${ }^{3}$ Hence $P_{i}$ is of height $n$ in $Z\left[X_{1}, \cdots, X_{n}\right]$. Since the Krull dimension of $Z\left[X_{1}, \cdots, X_{n}\right]$ is $n+1[2$, p. 28] and since $P$ has height $\geqq n+1$, it follows that $P$ is a maximal ideal of $Z\left[X_{1}, \cdots, X_{n}\right]$. Therefore $\sqrt{ }\left[A_{Z}+(p)\right]=\bigcap_{i=1}^{m} P_{i}^{\prime \prime}$, where each $P_{i}^{\prime \prime}$ is a maximal ideal. This implies that the ideal $A_{p}$ of $Z_{p}\left[X_{1}, \cdots, X_{n}\right]$ is of depth zero. By Lemma 2,

$$
\left|V_{\bar{z}_{p}}\left(A_{p}\right)\right|=\operatorname{dim}_{Z_{p}}\left\{Z_{p}\left[X_{1}, \cdots, X_{n}\right] /\left(\bigcap_{i=1}^{m} P_{i}^{\prime \prime}\right)\right\}
$$

and

$$
\left|V_{\bar{Q}}\left(A_{Q}\right)\right|=\operatorname{dim}_{Q}\left\{Q\left[X_{1}, \cdots, X_{n}\right] /\left(\bigcap_{i=1} P_{i}^{e}\right)\right\} .
$$

Thus the proof of the theorem will be complete if we show that if $\left\{\bar{h}_{1}^{p}, \cdots, \bar{h}_{v}^{p}\right\}$ is a basis for $Z_{p}\left[X_{1}, \cdots, X_{n}\right] /\left(\cap_{i=1}^{m} P_{i}^{\prime \prime}\right)$ over $Z_{p}$, then

\footnotetext{
${ }^{3}$ We use $P_{i}^{e}$ to denote the extension of $P_{i}$ to $Q\left[X_{1}, \cdots, X_{n}\right]$.
} 
the set of residue classes $\bar{h}_{i}$ determined by the $h_{i}$ 's in

$$
Q\left[X_{1}, \cdots, X_{n}\right] /\left(\bigcap_{i=1}^{s} P_{i}^{e}\right)
$$

is linearly independent over $Q$.

Suppose $\sum_{i=1}^{i}\left(a_{i} / b_{i}\right) \bar{h}_{i}=\overline{0}$, where $a_{i}, b_{i}$ are integers and $b_{i} \neq 0$ for each $i$. Then $\sum_{i=1}^{0}\left(a_{i} / b_{i}\right) h_{i} \in \bigcap_{i=1}^{s} P_{i}^{e}$ and if $m$ is the least common multiple of $b_{1}, \cdots, b_{v}$, then $\sum_{i=1}^{0}\left(a_{i} m / b_{i}\right) h_{i} \in \bigcap_{i=1}^{s} P_{i}$. If some $a_{i}$ is nonzero, let $d$ be the greatest common divisor of

$$
\left\{m a_{1} / b_{1}, \cdots, m a_{v} / b_{v}\right\} \text {. }
$$

Then $\sum_{i=1}^{0}\left(m a_{i} / b_{i}\right) h_{i}=d f$, where $f \in Z\left[X_{1}, \cdots, X_{n}\right]$. Since $\left(\bigcap_{i=1}^{s} P_{i}\right) \cap Z=(0)$, we see that $f \in \bigcap_{i=1}^{s} P_{i}$. Thus if $k_{i}=m a_{i} / b_{i}$ for $1 \leqq i \leqq v$, we may assume that $k_{1}, k_{2}, \cdots, k_{v}$ are relatively prime.

We have previously observed that each $P_{i}^{\prime \prime}$, for $i$ between 1 and $m$, contains some $P_{j}$, with $j$ between 1 and $s$. Hence it follows that $\bigcap_{i=1}^{s} P_{i} \subseteq \bigcap_{i=1}^{m} P_{i}^{\prime \prime}$. Thus $\sum_{i=1}^{0} k_{i} h_{i} \in \bigcap_{i=1}^{m} P_{i}^{\prime \prime}$, and the residue class modulo $p$ is zero. Thus since $\left\{\bar{h}_{i}^{p}\right\}_{i=1}^{0}$ is linearly independent over $Z_{p}$, we see that $p$ divides each $k_{i}$. This contradicts the assumption that $k_{1}, k_{2}, \cdots, k_{v}$ are relatively prime, and the proof of the theorem is complete.

In the above proof we proved that if certain primes $\left\{p_{i}^{\prime}\right\}$ were omitted, then Robinson's theorem was valid for all other primes $p$. The following example shows that each of the primes eliminated in the proof must be eliminated in order for Robinson's theorem to be valid. In this sense our proof produces the best possible collection of primes for which Robinson's theorem is valid.

Let $\left\{p_{i}^{\prime}\right\}_{i=1}^{n}$ be a finite set of $n$ distinct prime integers, and for each $i$ between 1 and $n$, let $f_{i}$ be the polynomial $p_{i}^{\prime} X_{i}$ of $Z\left[X_{1}, \cdots, X_{n}\right]$.

A shortest representation of the ideal $A_{z}$ generated by $\left\{f_{i}\right\}_{i=1}^{n}$ is $\bigcap_{i=0}^{n} P_{i}$, where $P_{0}=\left(X_{1}, \cdots, X_{n}\right), P_{1}=\left(p_{1}^{\prime}, X_{2}, \cdots, X_{n}\right), P_{2}$ $=\left(X_{1}, p_{2}^{\prime}, X_{3}, \cdots, X_{n}\right), \cdots, P_{n}=\left(X_{1}, \cdots, X_{n-1}, p_{n}^{\prime}\right)$, and each $P_{i}$ is prime in $Z\left[X_{1}, \cdots, X_{n}\right]$.

Note that $P_{0} \cap Z=(0)$, and for each $i$ between 1 and $n, P_{i} \cap Z$ $=\left(p_{i}^{\prime}\right) Z$. Also, the system of equations $\bar{f}_{i}^{p}=0$ has infinitely many solutions in any infinite field extension of $Z_{p}$ for $p=p_{i}^{\prime}$ for any $i$ between 1 and $n$.

\section{REFERENCES}

1. I. Kaplansky, Commutative rings, Allyn and Bacon, Boston, Mass., 1970. MR $40 \# 7234$. 
2. M. Nagata, Local rings, Interscience Tracts in Pure and Appl. Math., no. 13, Interscience, New York, 1962. MR 27 \#5790.

3. A. Rabinowitsch, Zum Hilbertschen Nullstellensatz, Math. Ann. 102 (1929), 520.

4. A. Robinson, Introduction to model theory and the metamathematics of algebra, North-Holland, Amsterdam, 1963. MR 26 \#4911.

5. B. L. van der Waerden, Moderne Algebra. Vol. 2, Springer, Berlin, 1940; English transl., Ungar, New York, 1950. MR 2, 120.

6. A. Weil, Foundations of algebraic geometry, rev. ed., Amer. Math. Soc. Colloq. Publ., vol. 29, Amer. Math. Soc., Providence, R.I., 1962. MR 26 \#2439.

7. O. Zariski, A new proof of Hilbert's Nullstellensatz, Bull. Amer. Math. Soc. 53 (1947), 362-368. MR 8, 499.

8. O. Zariski and P. Samuel, Commutative algebra. Vol. 2, University Series in Higher Math., Van Nostrand, Princeton, N.J., 1960. MR 22 \#11006.

Florida State University, Tallahassee, Florida 32306 